\title{
Astrophysical Bose-Einstein condensates and superradiance
}

\author{
Florian Kühnel ${ }^{1, *}$ and Cornelius Rampf ${ }^{2,3, \dagger}$ \\ ${ }^{1}$ Department of Physics, The Oskar Klein Centre for Cosmoparticle Physics, \\ Stockholm University, AlbaNova, 10691 Stockholm, Sweden \\ ${ }^{2}$ Institute of Cosmology and Gravitation, University of Portsmouth, \\ Portsmouth PO1 3FX, United Kingdom \\ ${ }^{3}$ Max-Planck-Institute for Gravitational Physics (Albert-Einstein-Institute), \\ 14476 Potsdam-Golm, Germany \\ (Received 9 September 2014; published 21 November 2014)
}

\begin{abstract}
We investigate gravitational analogue models to describe slowly rotating objects (e.g., dark-matter halos, or boson stars) in terms of Bose-Einstein condensates, trapped in their own gravitational potentials. We begin with a modified Gross-Pitaevskii equation, and show that the resulting background equations of motion are stable, as long as the rotational component is treated as a small perturbation. The dynamics of the fluctuations of the velocity potential are effectively governed by the Klein-Gordon equation of an "Eulerian metric," where we derive the latter by the use of a relativistic Lagrangian extrapolation. Superradiant scattering on such objects is studied. We derive conditions for its occurrence and estimate its strength. Our investigations might give an observational handle to phenomenologically constrain BoseEinstein condensates.
\end{abstract}

DOI: 10.1103/PhysRevD.90.103526

PACS numbers: 95.35.+d, 03.75.Kk, 95.30.Sf

\section{INTRODUCTION}

The history of superradiance possibly started with the discovery of the (inverse) Compton effect in 1923 by A. H. Compton [1], and has since then stimulated various works in different fields of physics and mathematics. The specific phenomenon of inertial superradiance due to the superluminal motion of a (charged) object through some medium goes back to investigations by P. A. Cherenkov in 1934 $[2,3]$. Since these pioneering works, which were both awarded with a Nobel Prize, the idea of rotational superradiance also arose, i.e., where a wave is inelastically scattered on a rotating rigid object such that its rotational momentum is transferred to the energy of the wave [4] (for an overview of superradiant phenomena see, e.g., Ref. [5]). In particular, investigations in the 70s then showed [6-9] that superradiance should also be spontaneously emitted by rotating black holes, where the space-time geometry can be described by a Kerr metric. Initially, the argument of superradiance was based on purely quantum-mechanical considerations, but it was Bekenstein in 1973 who realized that the argument should also hold in the classical sense to satisfy Hawking's classical horizon area theorem for black holes $[5,10]$.

Stimulated by these works, but also from the idea that black holes could radiate Hawking radiation [11], W. G. Unruh proposed in 1980 that the very occurrence of Hawking radiation is not related with generic properties of gravity, but just the result of evolving quantized

\footnotetext{
*florian.kuhnel@fysik.su.se

cornelius.rampf@port.ac.uk
}

perturbations at an (apparent) event horizon [12,13]. As a consequence, Hawking radiation should also become apparent at a sonic horizon when a fluid flow becomes transonic. The idea of analogue models of gravity, often dubbed acoustic black holes, was born. ${ }^{1}$ Since then, various efforts have been made to study Bose-Einstein condensates (BEC) in the laboratory to establish and to study analogue event horizons (cf. Refs. [14,15] and references therein).

Soon it became also clear that not only Hawking radiation but also superradiance should arise at acoustic black holes, simply because superradiance is the consequence of forming some horizon and an ergosphere [16,17] (provided that appropriate boundary conditions are established [18]), and thus has in general nothing to do with a space-time singularity, or with gravity in specific. Thus, analogous to Unruh's idea, the analogue scenario for extracting angular momentum from Kerr black holes would be a supersonic and a (mildly) rotating fluid flow.

In recent years, it has become very fruitful not only to study BECs in the laboratory but also to interpret macroscopic objects-such as (primordial) black holes [19-22], neutron/boson stars [23-25], white dwarfs [26], and/or darkmatter halos as BECs [27-29].

Here we follow a similar objective, although our approach is to some extent new. We shall begin with a modified Hamiltonian of the BEC with gravitational self-interactions, and we include a rotational kinetic term which is greatly suppressed with respect to its nonrotational kinetic term (see the following Sec. II). In Sec. III B, we then show that the resulting equations of motion are indeed stable, and we

\footnotetext{
${ }^{1}$ For a recent review on analogue gravity see Ref. [14].
} 
solve for the resulting density and velocity distribution of the BEC.

Generally, the inclusion of a rotational term in the Hamiltonian is known in studies related with generating vortex states in rapidly rotating BECs (cf. Refs. [30-33]), such as in superfluid helium II (cf. Refs. [34,35]), but we wish to stress again that we treat the rotational component as a small perturbation. As thoroughly explained in Sec. IV, the origin of the small "rotational" component arises from a relativistic coordinate transformation performed from a Lagrangian to an Eulerian/observer's frame. Actually, the fluid flow is still irrotational, but it is the fluid's space-time which is dragged with respect to the Eulerian frame. We analyze the properties of the Eulerian metric, which are somewhat similar to those of the Kerr metric, but we also refer to the Appendix for calculational details about its derivation. Equipped with the Eulerian metric, we solve its Klein-Gordon equation in Sec. V. Then, in Sec. VI, we derive the conditions for the occurrence of superradiance, and finally give a summary and outlook in Sec. VII.

\section{SYSTEM AND EQUATIONS OF MOTION}

Our starting point is a slowly rotating $d$-dimensional Bose-Einstein condensate in its own gravitational potential, being described by a complex scalar field $\hat{\psi}$ with the grand canonical Hamiltonian

$$
\begin{aligned}
\hat{\mathcal{H}}= & \int_{V} \mathrm{~d}^{d} x\left\{\hat{\psi}^{\dagger}\left[-\frac{1}{2 m} \Delta+\mu+\mathrm{i} \Omega \cdot(\boldsymbol{x} \times \nabla)\right] \hat{\psi}\right. \\
& \left.+\frac{1}{2} \int_{V} \mathrm{~d}^{d} y \hat{\psi}^{\dagger}(x) \hat{\psi}^{\dagger}(y) \frac{C}{|x-y|^{d-2}} \hat{\psi}(y) \hat{\psi}(x)\right\},
\end{aligned}
$$

where $\mu$ is the chemical potential and $V$ is the spatial volume of the halo. The third term on the right-hand side is due to a rotation around the axis $\Omega$. The condensate is supposed to be constituted by a number of $N \gg 1$ particles, and self-bound by a gravitational potential, with effective (attractive) interaction strength $C>0$. We set the Planck constant $\hbar$ equal to 1 , and express energy in units of the parameter $m$.

In Bose-Einstein condensates, the quantum state $\hat{\psi}$ consists of two components: a highly occupied groundstate, condensate part $\Psi:=\langle|\hat{\psi}|\rangle$, which shall here be described by a classical field ${ }^{2}$; and a quantum-fluctuation part $\hat{\phi}$, i.e., $\hat{\psi} \equiv \Psi+\hat{\phi}$. In the so-called Madelung representation [36], the condensate part reads

\footnotetext{
${ }^{2}$ We can describe the condensate part as a classical field because it is precisely a highly occupied state. This fact becomes clearer when expanding the field $\hat{\psi}$ into creation and annihilation operators, $\hat{a}^{\dagger}$ and $\hat{a}$, respectively. Due to the high occupation number $N \gg 1$, we have for the zero mode $\hat{a}_{\mathbf{k}=0}|N\rangle_{\mathrm{BEC}}=$ $\hat{a}_{\mathbf{k}=0} \sqrt{N}|N-1\rangle_{\mathrm{BEC}} \approx \sqrt{N}|N\rangle_{\mathrm{BEC}}$, which is tantamount to replacing $\hat{a}_{\mathbf{k}=0}$ with the $c$ number $\sqrt{N}$.
}

$$
\Psi \equiv \sqrt{n_{0}} \mathrm{e}^{\mathrm{i} S},
$$

with $n_{0}$ being the ground-state particle-number density, and the phase $S$ is the potential of the longitudinal part of the velocity.

We thus obtain a modified Gross-Pitaevskii equation (cf., e.g., Refs. [32,34,35])

$$
\mathrm{i} \partial_{t} \psi=\left[-\frac{1}{2 m} \Delta+\Xi+\mathrm{i} \Omega \cdot(\boldsymbol{x} \times \nabla)\right] \psi,
$$

where $\psi$ is the normalized wave function of the condensate, and $\Xi$ denotes the nonlocal gravitational potential induced through the condensate's density $\rho:=\psi^{\dagger} \psi$. In particular, the potential satisfies Poisson's equation

$$
\Delta \Xi=-\Omega_{d} C \rho,
$$

where $\Omega_{d}:=2 \pi^{d} / \Gamma(d / 2)$, and the latter is the gamma function. We obtain two independent real equations

$$
\begin{gathered}
\partial_{t} n_{0}+\nabla \cdot \boldsymbol{j}=\Omega \cdot\left(\boldsymbol{x} \times \nabla n_{0}\right), \\
\partial_{t} S-\frac{1}{2 m} \frac{1}{\sqrt{n_{0}}} \Delta \sqrt{n_{0}}+\frac{m}{2}(\nabla S)^{2}+\mu+\Xi \\
=m \Omega \cdot(\boldsymbol{x} \times \nabla S),
\end{gathered}
$$

with $\boldsymbol{j}:=n_{0} \nabla S$. The second term in Eq. (5b) is called the quantum potential. It has the dimension of energy, and it can be shown to be related to the trace of an intrinsically quantum-induced stress-energy tensor (cf. Ref. [14]). In the following, we will come back to this quantity, and derive a condition under which it can be neglected.

\section{STABILITY ANALYSIS}

To this end we expand the background particle-number density as well as the background phase up to first order, and furthermore treat the rotation terms as first-order perturbations. In doing so, we allow all perturbations to have a general coordinate dependence. This is important, because the system might be stable under perturbations that respect its symmetry, and unstable with regard to those that do not. We expand

$$
\begin{aligned}
& n(\boldsymbol{x}, t)=n_{0}(r)+\delta n(\boldsymbol{x}, t)+\text { higher orders, } \\
& S(\boldsymbol{x}, t)=S_{0}(r)+\delta S(\boldsymbol{x}, t)+\text { higher orders, } \\
& \Omega(\boldsymbol{x}, t)=\delta \Omega(\boldsymbol{x}, t)+\text { higher orders. }
\end{aligned}
$$

Note that we treat $\Omega$ as a small perturbation. This choice is not only important to stabilize our results, but we shall show that it is also physically motivated. 


\section{A. Background}

Here we consider the static case at the level of the background perturbations, and then add the perturbations in the stability analysis (see the following section). Equation (5a) is at the background level

$$
\nabla \cdot\left[n_{0} \nabla S_{0}\right] \simeq 0
$$

which yields the solution of the background fluid flow,

$$
\nabla S_{0}(r)=\frac{\boldsymbol{v}_{00}}{r^{d-1}} \frac{1}{n_{0}(r)}=: \boldsymbol{v}_{0} .
$$

Plugging this solution into Eq. (5b) and neglecting the quantum potential, we obtain an approximate expression for the chemical potential:

$$
\mu \simeq \frac{1}{2} m v_{00}^{2} \frac{1}{r^{2 d-2}} \frac{1}{n_{0}^{2}(r)}+C \int \mathrm{d}^{d} y \frac{n_{0}(|\boldsymbol{y}|)}{|\boldsymbol{x}-\boldsymbol{y}|^{d-2}},
$$

which we assume to be spatially constant, for simplicity. Then we obtain from the Laplacian of the above equation

$$
\bar{n}_{0}(r) \simeq \frac{1}{r^{d-1}} \partial_{r} r^{d-1} \partial_{r}\left[\frac{1}{r^{2 d-2}} \frac{1}{\bar{n}_{0}^{2}(r)}\right],
$$

where $\partial_{r}$ is the radial derivative in polar coordinates, and we have defined

$\bar{n}_{0}(r):=\frac{1}{\sqrt[3]{\bar{c}}} n_{0}(r), \quad \bar{c}:=\frac{1}{2} \bar{m} v_{00}^{2}, \quad \bar{m}:=\frac{m}{\Omega_{d} C}$.

The asymptotic behavior of the background solution is to a good approximation [22]

$$
\begin{aligned}
& n_{0}(r) \stackrel{r \rightarrow \infty}{\longrightarrow} \sim 1 / r^{d-1}, \\
& \boldsymbol{v}_{0}(r) \stackrel{r \rightarrow \infty}{\longrightarrow} \text { const. }
\end{aligned}
$$

To get a better intuition of the above, we solve numerically for $n_{0}(r)$ and $v_{0}(r)$ in the case of $d=3$; see Fig. 1. We have checked that the general behavior of $n_{0}(r)$ and $v_{0}(r)$ is essentially independent of the chosen initial conditions, which have to be imposed on Eqs. (9) and (10).

The above solutions for the background density and velocity have been derived under the assumption that the quantum pressure can be neglected. ${ }^{3}$ More precisely, validity requires

$$
\frac{1}{2 m} \frac{1}{\sqrt{n_{0}}} \Delta \sqrt{n_{0}} \simeq 0
$$

\footnotetext{
${ }^{3}$ This is commonly called the Thomas-Fermi approximation $[27,28,37]$.
}
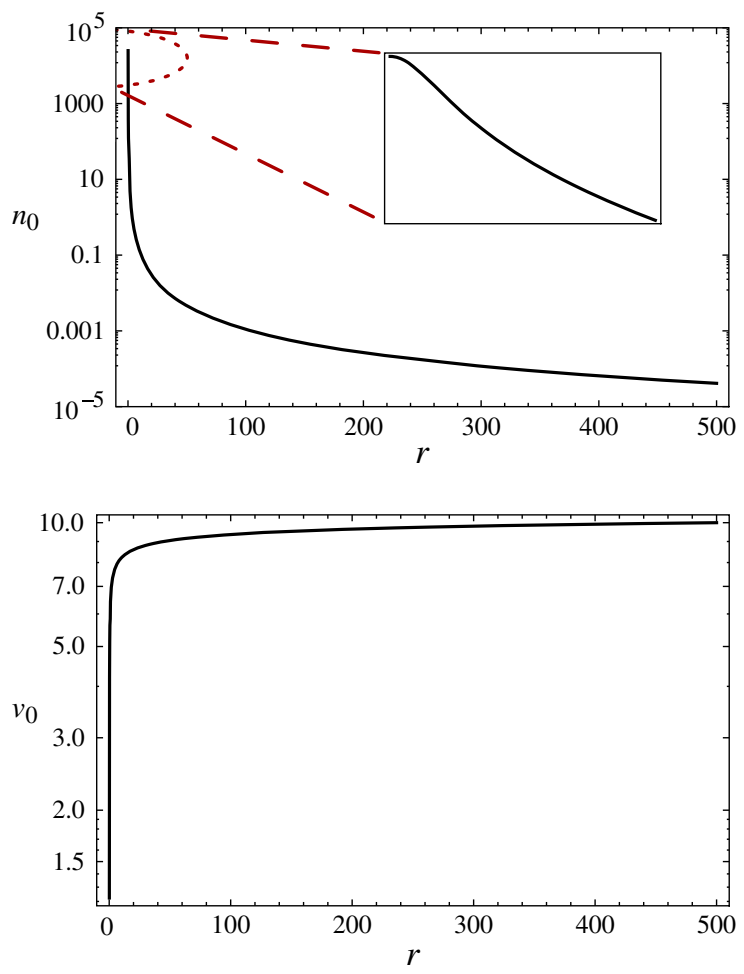

FIG. 1 (color online). Particle-number density $n_{0}(r)$ (left) and velocity $\left|v_{0}(r)\right|$ (right) for $d=3$ in units where $m=1$, and for $r_{\max }=500$ as well as $\left|v_{0}\left(r_{\max }\right)\right|=10$.

Now, in the outer region of a sufficiently large halo (in which we will be mainly interested), we have that the quantum pressure term actually diminishes approximately as $\sim r^{-2}$. This means that the quantity $x:=1 /\left(m r_{\max }^{2}\right)$ has to be sufficiently small. Equation (9) suggests that $x$, which has the dimension of energy, has to be compared to the chemical potential $\mu$. Thus, we get the approximate condition

$$
\mu / x=\mu m r_{\max }^{2} \gg 1,
$$

which translates to the condition for the maximal extent of the halo, i.e., $r_{\max } \gg \mu m^{-1 / 2}$.

\section{B. Stability}

We now perform a stability analysis. Using Ansätze (6a)-(6c), and the zeroth-order results for the velocity and density, Eqs. (8)-(10), we obtain up to first order

$\partial_{t} \delta n(\boldsymbol{x}, t) \simeq-\nabla \cdot\left[\nabla S_{0} \delta n+\frac{1}{m} n_{0} \nabla \delta S\right]+\Omega \cdot[\boldsymbol{x} \times \nabla \delta n]$,

$$
\begin{aligned}
\partial_{t} \delta S(\boldsymbol{x}, t) \simeq & -\nabla S_{0} \cdot \nabla \delta S+\Omega \cdot[\boldsymbol{x} \times \nabla \delta S] \\
& -\int \mathrm{d}^{d} y \frac{C}{|y-x|^{d-2}} \delta n(y, t),
\end{aligned}
$$


where we have suppressed some dependences for notational simplicity. In particular, $\Omega$ can vary with space and time. Acting with $\Delta$ on Eq. (15b), we find

$$
\delta n \simeq \frac{1}{\Omega_{d} C} \Delta\left(\partial_{t} \delta S+\nabla S_{0} \cdot \nabla \delta S-\Omega \cdot[x \times \nabla \delta S]\right) .
$$

Plugging Eq. (16) into Eq. (15a) yields

$$
\begin{gathered}
\Delta\left(\partial_{t}^{2} \delta S+\nabla S_{0} \cdot \nabla \partial_{t} \delta S-\Omega \cdot\left[\boldsymbol{x} \times \nabla \partial_{t} \delta S\right]\right) \\
\simeq-\nabla \cdot\left[\frac{\Omega_{d} C}{m} n_{0} \nabla \delta S+\nabla S_{0} \Delta\left(\partial_{t} \delta S\right.\right. \\
\left.\left.+\nabla S_{0} \cdot \nabla \delta S-\Omega \cdot\left[\boldsymbol{x} \times \nabla \partial_{t} \delta S\right]\right)\right] .
\end{gathered}
$$

Now, taking into account Eqs. (12a) and (12b), we are for large $r$ approximately left with

$$
\left[\partial_{t}+\nabla S_{0}(r) \cdot \nabla-\Omega(\boldsymbol{x}, t) \cdot(\boldsymbol{x} \times \nabla)\right]^{2} \delta \tilde{S}(\boldsymbol{x}, t) \simeq 0,
$$

where we have restored all dependences. The quantity $\delta \tilde{S}(\boldsymbol{x}, t):=\Delta \delta S(\boldsymbol{x}, t)$ can be thought of as the gradient of the velocity perturbation. Hence, any pulse keeps its shape at large distances from the center of the halo. Along similar lines, Eq. (15a) becomes

$\left[\partial_{t}+\nabla S_{0}(r) \cdot \nabla-\Omega(\boldsymbol{x}, t) \cdot(\boldsymbol{x} \times \nabla)\right] \delta n(\boldsymbol{x}, t) \simeq 0$.

Thus, our stability result also holds for arbitrary perturbations in the particle-number density. Moreover, these findings are true for all dimensions greater than two.

\section{EULERIAN METRIC}

As mentioned above we treat the rotation as a small perturbation with respect to the longitudinal velocity component. Here we include such a suppressed rotational velocity component by performing a gauge transformation of an irrotational fluid flow in a synchronous/comoving coordinate system to an observer's coordinate system. We call the synchronous/comoving coordinate systems the Lagrangian frame; we call the observer's coordinate system the Eulerian frame.

The essential idea of such a coordinate/gauge transformation is that its intrinsic nonlinear nature induces naturally a small amount of frame dragging [38]. Thus, even though the fluid motion is irrotational, the fluid's Lagrangian space-time is dragged with respect to the Eulerian frame. Such frame dragging is essential to observe superradiance.

For simplicity, we set $d=3$ from now on. We transform such an irrotational fluid flow to the Eulerian frame with the following line element (see the Appendix): $\mathrm{d} s^{2}=-\left(1-v_{\mathrm{L}}^{2}\right) \mathrm{d} t^{2}-2\left(\boldsymbol{v}_{\mathrm{L}}+\boldsymbol{v}_{\mathrm{T}}\right) \cdot \mathrm{d} \boldsymbol{x} \mathrm{d} t+\left(1-\frac{5}{2} \boldsymbol{v}_{\mathrm{L}}^{2}\right) \mathrm{d} \boldsymbol{x}^{2}$,

where $v_{\mathrm{L}}:=\nabla S_{0}$ and $\boldsymbol{v}_{\mathrm{T}}:=\nabla \times \boldsymbol{T}$ denote the longitudinal and a perturbatively suppressed transverse fluid velocity, respectively; $S_{0}$ is the background velocity potential, see Eq. (8). Calculational details about the transverse field $\boldsymbol{T}$ can be found in the Appendix, but we do not need its explicit form in the following, since we shall assume a constant amplitude in the latter, for simplicity. In the above line element, we have set the local speed of sound to 1, i.e., $c=1$. This approximation also means that we neglect pressure perturbations; this is consistent with the neglect of the quantum pressure term (see Sec. III A), but including pressure perturbations is far beyond the scope of this paper. We leave this issue for a forthcoming paper.

In this paper we assume that the longitudinal velocity component is much larger than the transverse component

$$
\frac{\left|v_{\mathrm{T}}\right|}{\left|\boldsymbol{v}_{\mathrm{L}}\right|} \ll 1
$$

It is difficult to estimate the precise amount of the suppression of the transverse velocity, since we heavily rely on a Lagrangian extrapolation far into the nonlinear regime. On cosmological scales, where the solutions are formally valid, we expect that the transverse velocity should be suppressed by a factor $\sim 10^{-3}-10^{-5}$. Note also that we can discard the term $\propto v_{\mathrm{L}}^{2}$ in the space-space component in Eq. (19) for sufficiently small velocities. ${ }^{4}$

Generally, the above line element is valid for an arbitrary geometry. In the following we consider an approximate spherical symmetry and also assume a stationary and convergent fluid flow [12]. Now, performing a temporal gauge transformation according to

$$
\tau(t, r)=t+\int^{r} \mathrm{~d} r^{\prime} \frac{v_{r}\left(r^{\prime}\right)}{1-v_{r}^{2}\left(r^{\prime}\right)}
$$

and setting $\boldsymbol{v}_{\mathrm{T}}:=\hat{\boldsymbol{e}}_{\phi} v_{\mathrm{T}}$, the metric (19) becomes

$$
\begin{aligned}
\mathrm{d} s^{2}= & -\left(1-v_{r}^{2}\right) \mathrm{d} \tau^{2}+\frac{1}{1-v_{r}^{2}} \mathrm{~d} r^{2}+r^{2} \mathrm{~d} \phi^{2}-2 r v_{\mathrm{T}} \mathrm{d} \phi \mathrm{d} \tau \\
& +2 r \frac{v_{r} v_{\mathrm{T}}}{1-v_{r}^{2}} \mathrm{~d} \phi \mathrm{d} r
\end{aligned}
$$

or, equivalently,

\footnotetext{
${ }^{4}$ Actually, the very term in the space-space component in Eq. (19) is of $\mathcal{O}\left(\boldsymbol{v}_{\mathrm{L}}^{4}\right)$, as can be seen when calculating the proper time between two events along the worldline [14,38].
} 


$$
\begin{aligned}
\mathrm{d} s^{2}= & -\left[1-\left(v_{r}^{2}+v_{\mathrm{T}}^{2}\right)\right] \mathrm{d} \tau^{2}+\frac{1}{1-v_{r}^{2}} \mathrm{~d} r^{2} \\
& +r^{2}\left(\mathrm{~d} \phi-\frac{v_{\mathrm{T}}}{r} \mathrm{~d} \tau\right)^{2}+2 r \frac{v_{r} v_{\mathrm{T}}}{1-v_{r}^{2}} \mathrm{~d} \phi \mathrm{d} r .
\end{aligned}
$$

Again, we have $v_{\mathrm{T}} / v_{r} \ll 1$, in accordance with the requirement (20). Note also that we have restricted the line element to the equatorial plane (i.e., $\mathrm{d} \theta=0$ ). Some properties of the above metric are identical to those of a Kerr metric, but it differs by one (i.e., the last) property:

(1) It is stationary, i.e., it does not explicitly depend on time.

(2) It is axisymmetric, i.e., it does not depend explicitly on $\phi$.

(3) It is not static, i.e., it is not invariant under time reversal $\tau \rightarrow-\tau$.

(4) It reduces to a Schwarzschild-like metric in the limit $v_{\mathrm{T}} \rightarrow 0$, if the fluid velocity smoothly exceeds the speed of sound $v_{r}:=1-\alpha(r-R)-\mathcal{O}\left(\alpha^{2}\right)$. This also means that we expect the occurrence of Hawking radiation [12,21] (see also Ref. [22]).

(5) There is a coordinate singularity when $v_{r} \rightarrow 1$ (i.e., when it approaches the local speed of sound), and a curvature singularity for $r \rightarrow 0$. Indeed, since $\lim _{r \rightarrow 0} v_{r} \propto 1 / r^{2}$, the 4-Ricci curvature is $\lim _{r \rightarrow 0}{ }^{(4)} R \propto$ $1 /\left[r^{6}\left(1+v_{\mathrm{T}}^{2}\right)\right]=\infty$.

(6) The metric is invariant under the simultaneous inversion $\tau \rightarrow-\tau, \phi \rightarrow-\phi .^{5}$

(7) The metric is not asymptotically flat, i.e., the metric does not reduce to the Minkowski metric in the limit $r \rightarrow \infty: \quad \mathrm{d} s^{2}=-C^{2} \mathrm{~d} \tau^{2}+C^{-2} \mathrm{~d} r^{2}+r^{2} \mathrm{~d} \phi^{2}-$ $2 r v_{\mathrm{T}} \mathrm{d} \phi \mathrm{d} \tau+2 r v_{\mathrm{T}} D \mathrm{~d} \phi \mathrm{d} r$, with $C=1-v_{r}^{2}=$ const, and $D=v_{r} C^{-2}=$ const.

Thus, because of the last property, the metric (23) is not identical to the Kerr metric. The above metric is not asymptotically flat due to the fact that the frame dragging is in principle apparent even at radial infinity, despite the fact that also the background density of the condensate is vanishing at infinity (see Fig. 1). This feature of the Eulerian metric is the result of the change of inertial frames (Mach's principle) between the condensate and the observer.
The occurrence of superradiance is not affected by the fact that our metric (23) is not asymptotically flat.

\section{KLEIN-GORDON EQUATION WITH TORTOISE COORDINATES}

Here we solve the relativistic Klein-Gordon equation for the linearized velocity-perturbation potential $\hat{\Phi} \equiv$ $1 /\left(2 m \mathrm{i} \sqrt{n_{0}}\right)\left(\mathrm{e}^{-\mathrm{i} S} \hat{\phi}+\mathrm{e}^{\mathrm{i} S} \hat{\phi}^{\dagger}\right)$, which reads (cf. Refs. [12,14])

$$
\partial_{\mu}\left(\sqrt{-g} g^{\mu \nu} \partial_{\nu}\right) \hat{\Phi}=0
$$

with $g=\operatorname{det} g_{\mu \nu}$, and summation over repeated space-time indices $\mu, \nu$ is implied. As mentioned earlier, we set $d=3$ for simplicity, although our calculations may be easily generalized to higher dimensions.

Note that we make use of two essential simplifications at this step. First, we suppress the "spatial dimension" related with the altitudinal angle $\mathrm{d} \theta$. Second, we assume that $v_{\mathrm{T}}$ is constant. Relaxing these approximations does not lead to any conceptual difficulties, but it would imply a dramatic increase in computational power. In this paper, we use these simplifying approximations mainly since we are only interested in the qualitative behavior of superradiance, and we leave more computationally demanding approaches for future work. Note, however, that $v_{\mathrm{T}}$ should be generally time dependent, since we expect that the negative momentum transfer due to superradiance leads to a decreasing $v_{\mathrm{T}}$ in time. However, considering the typically large amount of rotational energy as compared to that of the small amount of energy of a single scattered wave, it is a good approximation to neglect the time dependence of $v_{\mathrm{T}}$ for each individual scattered wave, which we do so in the following for simplicity.

Now we turn back to the Klein-Gordon Eq. (24). Let us therefore decompose the quantum field $\hat{\Phi}$ into creation and annihilation operators, $\hat{\alpha}^{\dagger}$ and $\hat{\alpha}$, respectively, i.e., $\hat{\Phi} \equiv \sum \hat{\alpha} f+$ H.c., for some mode function $f$. Then, using our result in Eq. (22), the Klein-Gordon equation becomes [39]

$$
\begin{aligned}
& r^{2}\left[1+v_{\mathrm{T}}^{2}-v_{r^{*}}^{2}\right] \Delta_{1}^{2} f_{, r^{*} r^{*}}-2 r v_{\mathrm{T}} v_{r^{*}} \Delta_{1} f_{, r^{*} \phi}+2 r\left[1+v_{\mathrm{T}}^{2}-v_{r^{*}}\left\{v_{r^{*}}+r \Delta_{1} v_{r^{*}, r^{*}}\right\}\right] \Delta_{1} f_{, r^{*}} \\
& \quad+\frac{r v_{\mathrm{T}}^{2}}{1-v_{r^{*}}^{2}}\left(\left[2 v_{r^{*}}+r \frac{1+v_{r^{*}}^{2}}{1-v_{r^{*}}^{2}} \Delta_{1} v_{r^{*}, r^{*}}\right] f_{, t}+r v_{r^{*}} \Delta_{1} f_{, t r^{*}}\right)-\frac{r^{2}\left(1-\left[1+v_{\mathrm{T}}^{2}\right] v_{r^{*}}^{2}\right)}{\left(1-v_{r^{*}}^{2}\right)^{2}} f_{, t t} \\
& \quad+f_{, \phi \phi}-v_{\mathrm{T}}\left(v_{r^{*}}+r \Delta_{1} v_{r^{*}, r^{*}}\right) f_{, \phi}-2 r \frac{v_{\mathrm{T}}}{1-v_{r^{*}}^{2}} f_{, t \phi}+\frac{r^{2} v_{\mathrm{T}}^{2} v_{r^{*}}}{1-v_{r^{*}}^{2}} \Delta_{1} f_{, t r^{*}}=0,
\end{aligned}
$$

where we have introduced the tortoise coordinates $r^{*}$, which are

\footnotetext{
${ }^{5}$ Note that the last term in (21) also flips the sign under the inversion $\tau \rightarrow-\tau$, as it should.
}

$$
\frac{\mathrm{d} r^{*}}{\mathrm{~d} r}=: \Delta_{1}, \quad \frac{\mathrm{d}}{\mathrm{d} r}=\Delta_{1} \frac{\mathrm{d}}{\mathrm{d} r^{*}}, \quad \Delta_{1}=\frac{1}{1-v_{r^{*}}^{2}} .
$$

$\Delta_{1}$ was derived from the requirement that the purely spatial and purely temporal parts of the metric (22) be conformally invariant, i.e., 


$$
X\left(-\mathrm{d} \tau^{2}+\mathrm{d} r^{* 2}\right) \stackrel{!}{=}-\left(1-v_{r^{*}}^{2}\right) \mathrm{d} \tau^{2}+\frac{1}{1-v_{r^{*}}^{2}} \mathrm{~d} r^{2},
$$

where $X$ is found to be $X=1 / \Delta_{1}$.

To solve the Klein-Gordon equation (25), we fix $v_{\mathrm{T}}$ to be a small ${ }^{6}$ constant, and we use the background solution of the longitudinal velocity $v(r) \simeq v_{0}(r)$, which is also depicted in the right panel of Fig. 1. Since the dependence of $\Delta_{1}$ (through $v_{r}$ ) on the tortoise coordinate $r^{*}$ is nontrivial, we numerically integrate

$$
r^{*}(r) \equiv \int \mathrm{d} r^{*}=\int \Delta_{1} \mathrm{~d} r
$$

and then use this result explicitly in the Klein-Gordon equation (25).

\section{SUPERRADIANCE IN THE FREQUENCY DOMAIN}

Using the tortoise coordinates from the previous section, and utilizing a decomposition of the mode function $f$ into base elements

$$
f\left(r^{*}, m, \omega\right)=z_{\omega, m}\left(r^{*}\right) \varphi_{\omega, m}\left(r^{*}\right) \mathrm{e}^{-\mathrm{i} m \phi} \mathrm{e}^{\mathrm{i} \omega t},
$$

we find, after plugging the above expression into Eq. (25), a second-order differential equation of the structure

$$
\left[\alpha_{2} \partial_{r^{*}}^{2}+\alpha_{1} \partial_{r^{*}}+\alpha_{0}\right]\left[z_{\omega, m}\left(r^{*}\right) \varphi_{\omega, m}\left(r^{*}\right)\right]=0,
$$

where $\alpha_{i} \equiv \alpha_{i}(\omega, m)$. We determine the function $z_{\omega, m}$ such that the coefficient of $\partial_{r^{*}} \varphi_{\omega, m}$ vanishes (cf. Ref. [39]). Then, the quasinormal form of the differential equation for $\varphi_{\omega, m}$ reads

$\partial_{r^{*}}^{2} \varphi_{\omega, m}\left(r^{*}\right)=-\left[\mathrm{V}\left(r^{*}, \omega, m\right)+\mathrm{i} \Gamma\left(r^{*}, \omega, m\right)\right] \varphi_{\omega, m}\left(r^{*}\right)$.

Here, both $\Gamma\left(r^{*}, \omega, m\right)$ and $\mathrm{V}\left(r^{*}, \omega, m\right)$ are real functions, and are given by

$$
\Gamma\left(r^{*}, \omega, m\right)=-\frac{m v_{\mathrm{T}} v_{r^{*}}^{3}\left(1-v_{r^{*}}^{2}\right)}{r^{* 2}\left(1-v_{r^{*}}^{2}+v_{\mathrm{T}}^{2}\right)},
$$

where we introduce the shorthand notation $v_{r^{*}} \equiv v_{0}\left(r^{*}\right)$ and we shall indicate spatial derivatives with respect to $r^{*}$ with a prime in the following - and

$$
\begin{aligned}
\mathrm{V}\left(r^{*}, \omega, m\right)= & \frac{1}{\left(r^{* 2}\left[1-v_{r^{*}}^{2}\right]^{2}\left[1+v_{\mathrm{T}}^{2}-v_{r^{*}}^{2}\right]^{2}\right)}\left[r^{*} v_{r^{*}}^{3}\left(r^{*} v_{\mathrm{T}}^{2}\left[2+v_{\mathrm{T}}^{2}\right] v_{r^{*}}^{\prime \prime}-2\left[4+3 v_{\mathrm{T}}^{2}\right] v_{r^{*}}^{\prime}\right)\right. \\
& +\left\{v_{\mathrm{T}}^{2}\left(r^{* 2} \omega^{2}-2 m r^{*} \omega v_{\mathrm{T}}-v_{\mathrm{T}}^{2}\right)-5\left[2+m^{2}\right]+\left(r^{* 2} \omega^{2}-2 m r^{*} \omega v_{\mathrm{T}}-\left[8+5 m^{2}\right] v_{\mathrm{T}}^{2}\right)\right\} v_{r^{*}}^{8} \\
& +\left(\left[5+m^{2}\right]+\left[2+m^{2}\right] v_{\mathrm{T}}^{2}\right) v_{r^{*}}^{10}-v_{r^{*}}^{12}-2 r^{*}\left[4+v_{\mathrm{T}}^{2}\right] v_{r^{*}}^{7} v_{r^{*}}^{\prime}+2 r^{*} v_{r^{*}}^{9} v_{r^{*}}^{\prime} \\
& +\left[1+v_{\mathrm{T}}^{2}\right] v_{r^{*}}^{2}\left\{\left(1+5 m^{2}-4 r^{* 2} \omega^{2}+8 m r^{*} \omega v_{\mathrm{T}}+v_{\mathrm{T}}^{2}\right)-2 r^{* 2} v_{\mathrm{T}}^{2} v_{r^{*}}^{\prime 2}\right\} \\
& -\left[1+v_{\mathrm{T}}^{2}\right]\left\{\left(m^{2}-r^{*} \omega\left[r^{*} \omega-2 m v_{\mathrm{T}}\right]\right)+r^{* 2} v_{\mathrm{T}}^{2} v_{r^{*}}^{\prime 2}\right\} \\
& +v_{r^{*}}^{4}\left\{\left[1+v_{\mathrm{T}}^{2}\right]\left(6 r^{* 2} \omega^{2}-5\left[1+2 m^{2}\right]-3 v_{\mathrm{T}}\left[4 m r^{*} \omega+v_{\mathrm{T}}\right]\right)+3 r^{* 2} v_{\mathrm{T}}^{2} v_{r^{*}}^{\prime 2}\right\} \\
& +\left\{10\left[1+m^{2}\right]-v_{\mathrm{T}}^{2}\left(4 r^{* 2} \omega^{2}-8 m r^{*} \omega v_{\mathrm{T}}-3 v_{\mathrm{T}}^{2}\right)-2\left(2 r^{* 2} \omega^{2}-4 m r^{*} \omega v_{\mathrm{T}}-\left[6+5 m^{2}\right] v_{\mathrm{T}}^{2}\right)\right\} v_{r^{*}}^{6} \\
& \left.+r^{*} v_{r^{*}}^{5}\left(6\left[2+v_{\mathrm{T}}^{2}\right] v_{r^{*}}^{\prime}-r^{*} v_{\mathrm{T}}^{2} v_{r^{*}}^{\prime \prime}\right)+r\left[1+v_{\mathrm{T}}^{2}\right] v_{r^{*}}\left(2 v_{r^{*}}^{\prime}-r^{*} v_{\mathrm{T}}^{2} v_{r^{*}}^{\prime \prime}\right)\right] .
\end{aligned}
$$

Now, as we have seen in Sec. III A, the background velocity approaches a constant for large $r^{*}$. In this limit we find

$$
r^{*} \Gamma\left(r^{*}, \omega, m\right) \simeq 0, \quad \mathrm{~V}\left(r^{*}, \omega, m\right) \simeq \tilde{\omega}^{2},
$$

where

$$
\tilde{\omega} \simeq \omega \frac{\left(1-v_{r^{*}}^{2}\right) \sqrt{1+v_{\mathrm{T}}^{2}}}{1-v_{r^{*}}^{2}+v_{\mathrm{T}}^{2}} .
$$

Note that $\lim _{v_{T} \rightarrow 0} \tilde{\omega}=\omega$, so the energy of the outgoing wave is identical to the energy of the ingoing wave in this

\footnotetext{
${ }^{6} v_{\mathrm{T}}$ has to be small because of the requirement from (20), which itself is based on the Appendix.
}

limit. The above relation also means that, for large $r^{*}$, the solution of Eq. (31) for $\varphi \equiv \varphi_{\omega, m}$ can be written as

$$
\varphi\left(r^{*}\right) \simeq \mathrm{e}^{-\mathrm{i} \tilde{\omega} r^{*}}+\mathcal{R} \mathrm{e}^{\mathrm{i} \tilde{\omega} r^{*}}, \quad r^{*} \rightarrow \infty,
$$

which consists of an incident wave of unit amplitude and a scattered one with an amplitude given by the reflection coefficient $\mathcal{R}$. The extraction of (the condensate's rotational) energy by a scattered wave is one example of the phenomenon of superradiance (cf. Ref. [5] for a review). It occurs if ${ }^{7}$

\footnotetext{
${ }^{7}$ This condition can be shown to be equivalent to that of an imaginary Wronskian (cf. Ref. [18]).
} 


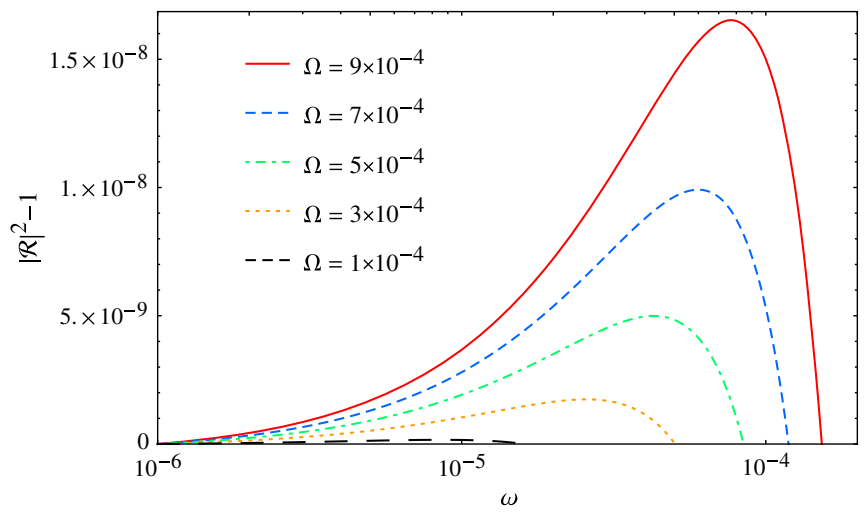

FIG. 2 (color online). $|\mathcal{R}|^{2}-1$ as a function of frequency for $d=3$ and for various values of $\Omega:=v_{\mathrm{T}} /\left|\boldsymbol{v}_{0}\left(r_{\max }^{*}\right)\right|$. The other parameters are $m=1, r_{\max }^{*}=500$ and $\left|v_{0}\left(r_{\max }^{*}\right)\right|=10$.

$$
|\mathcal{R}|^{2}>1
$$

In Fig. 2, we show precisely this quantity for the most relevant case of three spatial dimensions, and for various small values of the ratio $\Omega:=v_{\mathrm{T}} /\left|\boldsymbol{v}_{0}\left(r_{\max }^{*}\right)\right|$ for which our approximation is valid [cf. Eq. (20) and also the Appendix]. We observe that the system becomes more and more superradiant if this ratio is increased. This can be achieved either by increasing the rotation of the object, or by decreasing the (outer) radial velocity. However, for all studied parameter values $\Omega$, the corresponding amplification is quite small.

We find that, as expected, superradiance is only present for small enough frequencies, i.e., for $\omega<\omega_{\max }$. Here, $\omega_{\max }>0$ is defined by the condition $\left|\mathcal{R}\left(\omega_{\max }\right)\right|^{2} \stackrel{!}{=} 1$. For the present choice of parameters $\left(r_{\max }^{*}=500\right.$ and $\left|v_{0}\left(r_{\max }^{*}\right)\right|=$ $10)$, we obtain for all studied curves the fixed "law" of superradiance, which is to a very good approximation

$$
\omega<0.02 m \Omega,
$$

which is our central result in this section.

\section{SUMMARY AND OUTLOOK}

In this work we have investigated stability and dynamical properties of slowly rotating gravitationally self-bound Bose-Einstein condensates. Although the model under consideration is rather general, we primarily focused on respective dark-matter halos. However, we expect that our considerations should also hold for Bose-Einstein condensates of white dwarfs, neutron and boson stars.

First, we derived the modified Gross-Pitaevskii equation and found general solutions for the background particlenumber density and proved that the associated background equations of motion are stable in all dimensions. This holds true as long as the rotational component is treated as a small perturbation. We showed that the dynamics of the perturbation of the velocity potential are effectively governed by the Klein-Gordon equation of a newly derived "Eulerian metric."

The latter is based on a relativistic coordinate transformation from the (Lagrangian) fluid frame to an observer's frame, where a small "rotational" component naturally arises because of the inherent nonlinearities due to the gravitational instability. Physically, the fluid flow is irrotational, but the Eulerian frame is dragged with respect to the Lagrangian frame, where the fluid is at rest. We thus associate the small rotational component with Lagrangian transverse fields, which manifests in a frame dragging. We would like to emphasize that the derived Eulerian metric is generally valid on cosmological scales, and that the reported geometrical correspondence with that of the Bose-Einstein condensate relies heavily on a Lagrangian extrapolation, i.e., the correspondence holds only approximately.

We then analyzed the properties of this Eulerian metric, which shares many properties of the Kerr metric (e.g., the Eulerian metric has an event horizon and an ergosphere, when the fluid velocity becomes transonic), but differs in that the Eulerian metric is not asymptotically flat. (The latter has no consequence for the occurence of superradiance.)

Then, we particularly investigated the effect of superradiance at the vicinity of Bose-Einstein condensates. For convenience, we restricted our investigation to a $(2+1)$ dimensional description [16,39]. Our formalism has the advantage that the boundary conditions of the velocity and density are everywhere well defined and, in particular, our model does not possess a divergent velocity at the origin, as is the case in the draining bathtub model. We found that superradiance actually does occur, and computed the precise form of the reflection coefficient. We found that, in our general model, amplification takes place when $\omega<$ $\omega_{\max }=0.02 \mathrm{~m} \Omega$ is satisfied, where $\Omega$ is the ratio of transverse and longitudinal velocity of the condensate, and $m$ is the azimuthal quantum number of the scattered wave.

As halos typically rotate quite slowly (cf. Ref. [40]), the energy gain of these waves is naturally rather small. Note that such a suppressed rotation is actually a prediction of our model. The precise level of suppression can vary from model to model, so we depicted in Fig. 2 various values for $\Omega$, ranging from $10^{-3}-10^{-4}$. From the results, we indeed conclude that the more rapidly the condensate rotates, the more superradiance is expected, and the larger is the maximum frequency below which amplification takes place.

Our results may provide an observational tool to discriminate/constrain possible Bose-Einstein condensate dark-matter halo models. In particular, radiation from intense sources such as supernovae or gamma-ray bursts in the line of sight behind a possible dark-matter halo 
will be an optimal test for this class of models. Another set of constraints might come from gravitational lensing, which is expected to be larger than in the conventional cases (cf., e.g., Ref. [41] for Bose stars, and Ref. [42] for scalar-field halos). Of course, similar investigations could be applied to other astrophysical condensates.

\section{ACKNOWLEDGMENTS}

C. R. acknowledges the support of individual fellowship No. RA 2523/1-1 from the German research organization (DFG). F. K. was supported by the Swedish Research Council (VR) through the Oskar Klein Centre. C. R. thanks the Albert Einstein Institute (Potsdam/Golm) for its hospitality, particularly Lars Andersson. F. K. would like to thank the Institute of Gravitation and Cosmology at the University of Portsmouth for hospitality.

\section{APPENDIX: DERIVATION OF THE EULERIAN LINE ELEMENT [EQ. (19)]}

In this Appendix, we show how to obtain the line element (19), i.e., the Eulerian metric which results from a relativistic Lagrangian extrapolation of an approximately spherical overdensity in the deeply nonlinear regime. We use the relativistic Lagrangian perturbation theory up to second order [38] to make the nonlinear frame dragging in the line element apparent. Note explicitly that we assume a nonexact spherical overdensity; if we had assumed an exact spherical symmetry, the second-order terms would be exactly zero.

Before proceeding, we wish to summarize the calculational steps. First, we perform a specific gauge transformation from the synchronous/comoving gauge (the Lagrangian frame) to the Eulerian gauge. ${ }^{8}$ The corresponding Eulerian line element will have no specified symmetry yet. By restricting the metric to an approximate spherical symmetry, we shall show that the Eulerian metric reduces to a Kerr-like metric (being, however, not asymptotically flat).

The synchronous/comoving line element is (summation over repeated indices is assumed)

$$
\mathrm{d} s^{2}=-\mathrm{d} t^{2}+a^{2}(t) \gamma_{i j}(t, \boldsymbol{q}) \mathrm{d} q^{i} \mathrm{~d} q^{j}
$$

with the following solution for an irrotational cold darkmatter component:

\footnotetext{
${ }^{8}$ In the nonperturbative treatment, the Eulerian gauge can be understood in terms of a simple ADM shift, where the shift is directly associated with the Lagrangian displacement field [43].
}

$$
\begin{aligned}
\gamma_{i j}(t, \boldsymbol{q})= & \delta_{i j}\left(1+\frac{10}{3} \varphi\right)+3 a(t) t_{0}^{2}\left[\varphi_{, i j}\left(1-\frac{10}{3} \varphi\right)\right. \\
& \left.-5 \varphi_{, i} \varphi_{, j}+\frac{5}{6} \delta_{i j} \varphi_{, l} \varphi^{, l}\right] \\
& -\left(\frac{3}{2}\right)^{2} \frac{3}{7} a^{2}(t) t_{0}^{4}\left[4 \varphi_{, i j} \nabla_{q}^{2} \varphi-2 \delta_{i j} \mu_{2}\right] \\
& +\left(\frac{3}{2}\right)^{2} \frac{19}{7} a^{2}(t) t_{0}^{4} \varphi_{, l i} \varphi_{, j}^{l,},
\end{aligned}
$$

where we have defined $\mu_{2}:=1 / 2\left[\left(\nabla_{q}^{2} \varphi\right)^{2}-\varphi_{, l m} \varphi^{, l m}\right] .{ }^{9}$ For the sake of generality, we include in this Appendix also the cosmological scale factor $a(t)$; for our specific case in the main text, $a$ can be viewed as a perturbation parameter (which we finally suppress in the main text). $\varphi$ denotes the cosmological potential, i.e., some initial condition (in our case, with an approximately spherical symmetry) normalized at some initial time $t_{0}$. The above solution can be derived by using standard cosmological perturbation theory [44], the gradient expansion technique [45,46], or the tetrad formalism [47], and is valid for an Einstein-de Sitter (EdS) universe. ${ }^{10}$ We define the Eulerian gauge by

$\mathrm{d} s^{2}=-(1+2 A) \mathrm{d} t^{2}+2 a w_{i} \mathrm{~d} t \mathrm{~d} x^{i}+a^{2} G_{i j} \mathrm{~d} x^{i} \mathrm{~d} x^{j}$,

with the spatial metric $G_{i j}=\delta_{i j}[1-2 B]$, where $A$ and $B$ are scalar perturbations, and, as we shall see, $\boldsymbol{w}$ contains a solenoidal and a vector part (in the nonperturbative treatment, $\boldsymbol{w}$ is just the ADM shift). We neglect tensor perturbations, as they are of no importance in what follows.

The coordinate transformation is

$$
x^{\mu}(t, \boldsymbol{q})=q^{\mu}+F^{\mu}(t, \boldsymbol{q}),
$$

with

$x^{\mu}=\left(\begin{array}{l}t \\ \boldsymbol{x}\end{array}\right), \quad q^{\mu}=\left(\begin{array}{l}t \\ \boldsymbol{q}\end{array}\right), \quad$ and $\quad F^{\mu}=\left(\begin{array}{l}0 \\ \boldsymbol{F}\end{array}\right)$,

where $x^{\mu}$ are the coordinates in the Eulerian line element (A3), and $q^{\mu}$ is the one in the synchronous/comoving line element. Explicitly, the time coordinates in both coordinate systems are identical. Thus, the above is a purely spatial gauge transformation. The relation of the metrics is

$$
g_{\mu \nu}(t, \boldsymbol{q})=\frac{\partial x^{\tilde{\mu}}}{\partial q^{\mu}} \frac{\partial x^{\tilde{\nu}}}{\partial q^{\nu}} g_{\tilde{\mu} \tilde{\nu}}(\tau, \boldsymbol{x}) .
$$

The metric coefficients in the Eulerian gauge in (A3), $\{A, B, \boldsymbol{w}\}$, are unknown, and we shall calculate them up

\footnotetext{
${ }^{9}$ Here, indices are lowered and raised by the use of the Kronecker delta.

${ }^{10}$ Actually, the solutions for the Eulerian gauge hold also for a $\Lambda \mathrm{CDM}$ universe, with only minor modifications in the time evolution coefficients [43].
} 
to $\mathcal{O}\left(\varphi^{2}\right)$ by using the above relation. Truncating up to second order, the resulting relations between the spacespace, space-time, and time-time parts of the metrics are, respectively,

$$
\gamma_{i j}(t, \boldsymbol{q}) \simeq \delta_{i j}[1-2 B(t, \boldsymbol{x})]+2 F_{(i, j)}(t, \boldsymbol{q})(1-2 B)+F_{l, i} F_{l, j},
$$

$0 \simeq a^{2}[1-2 B] \frac{\partial F_{i}(t, \boldsymbol{q})}{\partial t}+a^{2} F_{l, i} \frac{\partial F_{l}}{\partial t}+a w_{i}(\tau, \boldsymbol{x})+a w_{l} F_{l, i}$,

$$
-1 \simeq-1-2 A(\tau, \boldsymbol{x})+2 a w_{l} \frac{\partial F_{l}}{\partial t}+a^{2} \frac{\partial F_{l}}{\partial t} \frac{\partial F_{l}}{\partial t} .
$$

Solving Eqs. (A7a)-(A7c) with an iterative technique, we obtain for the gauge generator, i.e., the 3-displacement field up to second order,

$$
\begin{aligned}
F_{i}(t, \boldsymbol{q})= & \frac{3}{2} a t_{0}^{2} \varphi_{, i}(\boldsymbol{q})-\left(\frac{3}{2}\right)^{2} \frac{3}{7} a^{2} t_{0}^{4} \frac{\partial_{i}}{\nabla^{2}} \mu_{2} \\
& +5 a t_{0}^{2}\left(\partial_{i} C-\partial_{i} \varphi^{2}+R_{i}\right)
\end{aligned}
$$

where we have defined the two terms

$$
\begin{aligned}
& C=\frac{1}{\nabla^{2} \nabla^{2}}\left[\frac{3}{4} \varphi_{, l l} \varphi_{, m m}+\varphi_{, l} \varphi_{, l m m}+\frac{1}{4} \varphi_{, l m} \varphi_{, l m}\right], \quad \text { (A9a) } \\
& R_{i}=\frac{1}{\nabla^{2} \nabla^{2}}\left[\varphi_{, i l} \varphi_{, m m l}-\varphi_{, l l i} \varphi_{, m m}+\varphi_{, i} \varphi_{, l l m m}-\varphi_{, m} \varphi_{, m l l i}\right] .
\end{aligned}
$$

The first term denotes a purely longitudinal contribution; the latter term denotes a purely transverse contribution to the displacement, and thus to the velocity field as well. Both terms are of purely relativistic origin. For the perturbations in the Eulerian line element, we obtain

$$
\begin{aligned}
A(t, \boldsymbol{x}) & =-\frac{1}{2} a t_{0}^{2} \varphi_{, l} \varphi^{l}, \\
B(t, \boldsymbol{x}) & =-\frac{5}{3} \varphi(\boldsymbol{x})+\frac{5}{2} a t_{0}^{2}\left[\frac{1}{\nabla_{x}^{2}} \mu_{2}+\frac{1}{2} \varphi_{, l} \varphi^{, l}\right], \\
a w_{i}(t, \boldsymbol{x}) & =-\mathcal{S}_{, i}^{\mathrm{N}}+\partial_{i}\left[\frac{5}{3} t \varphi^{2}-\frac{10}{3} t C\right]-\frac{10}{3} t R_{i},
\end{aligned}
$$

with

$$
\mathcal{S}^{\mathrm{N}}=\varphi(\boldsymbol{x}) t-\frac{3}{2} t_{0}^{4 / 3} t^{5 / 3} \frac{1}{\nabla_{\boldsymbol{x}}^{2}} G_{2}(\boldsymbol{x})
$$

$$
G_{2}=\frac{3}{7}\left(\nabla_{x}^{2} \varphi\right)^{2}+\varphi_{, l} \nabla_{x}^{2} \varphi^{l}+\frac{4}{7} \varphi_{, l m} \varphi^{, l m},
$$

where $G_{2}$ is the well-known second-order EdS kernel for the velocity field at second order in Newtonian perturbation theory [48]. The term $A$ is the (linear) velocity of the fluid squared (generally contracted with the spatial metric which is, however, a third-order effect), thus denotes nothing but the Lorentz boost from special relativity. Indeed, $A$ can be written in terms of the time derivative of the displacement $\boldsymbol{F}$, as can be easily proven, $A \simeq-1 / 2 a^{2}(\partial \boldsymbol{F} / \partial t)^{2}$. The scalar $B$ contains the linear initial conditions [first term in Eq. (A10b)] and some relativistic corrections. Since we are only interested here in dynamical effects, we neglect the 3-curvature in this paper and thus set the spatial 3-metric components to unity, $G_{i j} \rightarrow \delta_{i j}$ (cf. the Minkowskian limit defined in Ref. [49]). The quantity $\boldsymbol{w}$ [see Eq. (A10c)] contains the velocity information of the fluid: $w_{i}=-G_{i j} a \partial F^{i} / \partial t$, where the spatial dependence on the right-hand side is with respect to the Eulerian coordinates $(t, \boldsymbol{x}) \cdot{ }^{11}$ Note explicitly that $\boldsymbol{w}$ contains not only a longitudinal velocity but also a small transverse component, cf. the term $\boldsymbol{R}$ in Eq. (A10c), while the metric component $A$ only contains a longitudinal velocity component, see Eq. (A10a). In fact, $A$ remains purely longitudinal up to the third order, so the transverse contribution in $A$ is suppressed by a factor $\varphi^{2}$.

Because of the these considerations, we write $A=-1 / 2 v_{\mathrm{L}}^{2}$, $B=5 / 4 v_{\mathrm{L}}^{2}$, and $-\boldsymbol{w}=\boldsymbol{v}_{\mathrm{L}}+\boldsymbol{v}_{\mathrm{T}}$, where $\boldsymbol{v}_{\mathrm{L}}$ denotes the longitudinal part of the velocity, and the transverse velocity $v_{\mathrm{T}}$ is suppressed by only one $\varphi$ with respect to $\boldsymbol{v}_{\mathrm{T}}{ }^{12}$ Note that we have absorbed a scale factor in the definition in the velocities. To derive the Eulerian metric for an approximate spherical symmetry, one then has to assume

$$
\varphi(\boldsymbol{x}) \simeq \varphi(r)+\epsilon^{1 / 2} \varphi_{\Omega},
$$

where the first term denotes the symmetric contribution, and the latter term a small perturbation along some given axis $\Omega$. The Eulerian metric is then

$$
\begin{aligned}
\mathrm{d} s^{2} \simeq & -\left(1-\boldsymbol{v}_{\mathrm{L}}^{2}\right) \mathrm{d} t^{2}-2\left(\boldsymbol{v}_{\mathrm{L}}+\boldsymbol{v}_{\mathrm{T}}\right) \cdot \mathrm{d} \boldsymbol{x} \mathrm{d} t \\
+ & {\left[1-\frac{5}{2} \boldsymbol{v}_{\mathrm{L}}^{2}\right] \mathrm{d} \boldsymbol{x} \cdot \mathrm{d} \boldsymbol{x} . }
\end{aligned}
$$

Note that the above relies on a Lagrangian extrapolation far into the nonlinear regime, and we have nelgected the curvature metric $G_{i j} \rightarrow \delta_{i j}$. Also note that this extrapolation is formally valid for any seed with arbitrary $\varphi$.

\footnotetext{
${ }^{12}$ The transverse velocity $\boldsymbol{v}_{\mathrm{T}}$ is proportional to $\boldsymbol{R}$.
} 
[1] A. H. Compton, Phys. Rev. 21, 483 (1923).

[2] I. M. Frank and I. Tamm, C.R. Hebd. Seances Acad. Sci. 14, 109 (1937).

[3] V. L. Ginzburg and I. M. Frank, Zh. Eksp. Teor. Fiz. 16, 15 (1946) [J. Phys. (USSR) 9, 353 (1945)].

[4] R. H. Dicke, Phys. Rev. 93, 99 (1954).

[5] J. D. Bekenstein and M. Schiffer, Phys. Rev. D 58, 064014 (1998).

[6] Ya. B. Zel'dovich, Zh. Eksp. Teor. Fiz. 14, 270 (1971) [JETP Lett. 14, 180 (1971)].

[7] Ya. B. Zel'dovich, Sov. Phys. JETP 35, 1085 (1972).

[8] C. W. Misner, Phys. Rev. Lett. 28, 994 (1972).

[9] W. G. Unruh, Phys. Rev. D 10, 3194 (1974).

[10] J. D. Bekenstein, Phys. Rev. D 7, 949 (1973).

[11] S. W. Hawking, Commun. Math. Phys. 43, 199 (1975); 46, 206 (1976).

[12] W. G. Unruh, Phys. Rev. Lett. 46, 1351 (1981).

[13] M. Visser, Int. J. Mod. Phys. D 12, 649 (2003).

[14] C. Barcelo, S. Liberati, and M. Visser, Living Rev. Relativity 8, 12 (2005); 14, 3 (2011).

[15] C. Barcelo, S. Liberati, and M. Visser, Int. J. Mod. Phys. A 18, 3735 (2003).

[16] S. Basak and P. Majumdar, Classical Quantum Gravity 20, 3907 (2003).

[17] S. Basak and P. Majumdar, Classical Quantum Gravity 20, 2929 (2003).

[18] M. Richartz, S. Weinfurtner, A. J. Penner, and W. G. Unruh, Phys. Rev. D 80, 124016 (2009).

[19] M. Khlopov, B. A. Malomed, and I. B. Zeldovich, Mon. Not. R. Astron. Soc. 215, 575 (1985).

[20] G. Dvali and C. Gomez, Fortschr. Phys. 61, 742 (2013).

[21] G. Dvali and C. Gomez, Eur. Phys. J. C 74, 2752 (2014).

[22] F. Kuhnel, Phys. Rev. D 90, 084024 (2014).

[23] C. Gruber and A. Pelster, arXiv:1403.3812.

[24] X. Z. Wang, Phys. Rev. D 64, 124009 (2001).

[25] B. Kleihaus, J. Kunz, and S. Schneider, Phys. Rev. D 85, 024045 (2012).

[26] O. G. Benvenuto and M. A. De Vito, J. Cosmol. Astropart. Phys. 02 (2011) 033.
[27] C. G. Boehmer and T. Harko, J. Cosmol. Astropart. Phys. 06 (2007) 025.

[28] P. Sikivie and Q. Yang, Phys. Rev. Lett. 103, 111301 (2009).

[29] P.-H. Chavanis, Phys. Rev. D 84, 063518 (2011).

[30] R. P. Yu and M. J. Morgan, Classical Quantum Gravity 19, L157 (2002).

[31] N. T. Zinner, Phys. Res. Int. 2011, 734543 (2011).

[32] T. Rindler-Daller and P. R. Shapiro, Mon. Not. R. Astron. Soc. 422, 135 (2012).

[33] F. Federici, C. Cherubini, S. Succi, and M. P. Tosi, Phys. Rev. A 73, 033604 (2006).

[34] S. Sinha and Y. Castin, Phys. Rev. Lett. 87, 190402 (2001).

[35] U. R. Fischer and G. Baym, Phys. Rev. Lett. 90, 140402 (2003).

[36] E. Madelung, Z. Phys. 40, 322 (1927).

[37] F. Dalfovo, S. Giorgini, L. P. Pitaevskii, and S. Stringari, Rev. Mod. Phys. 71, 463 (1999).

[38] C. Rampf, Phys. Rev. D 89, 063509 (2014).

[39] E. Berti, V. Cardoso, and J. P. S. Lemos, Phys. Rev. D 70, 124006 (2004).

[40] P. Bett, V. Eke, C. S. Frenk, A. Jenkins, and T. Okamoto, Mon. Not. R. Astron. Soc. 404, 1137 (2010).

[41] M. P. Dabrowski and F. E. Schunck, Astrophys. J. 535, 316 (2000).

[42] F. E. Schunck, B. Fuchs, and E. W. Mielke, Mon. Not. R. Astron. Soc. 369, 485 (2006).

[43] C. Rampf and A. Wiegand, arXiv:1409.2688 [Phys. Rev. D (to be published)].

[44] S. Matarrese, S. Mollerach, and M. Bruni, Phys. Rev. D 58, 043504 (1998).

[45] C. Rampf and G. Rigopoulos, Phys. Rev. D 87, 123525 (2013).

[46] C. Rampf and G. Rigopoulos, Mon. Not. R. Astron. Soc. Lett. 430, L54 (2013).

[47] H. Russ, M. Morita, M. Kasai, and G. Borner, Phys. Rev. D 53, 6881 (1996).

[48] F. Bernardeau, S. Colombi, E. Gaztanaga, and R. Scoccimarro, Phys. Rep. 367, 1 (2002).

[49] T. Buchert and M. Ostermann, Phys. Rev. D 86, 023520 (2012). 